\title{
Application Effect of Time Nursing Theory Based Clinical Nursing Pathway on Gestational Hypertension Patients
}

\author{
Y. ZHOU, Z. JI*, R. DU, G. M. RAO ${ }^{1}$ AND Y. N. ZHAO \\ Department of Cardiovascular Medicine, ${ }^{1}$ Department of Neurology, Tangshan Gongren Hospital, Tangshan 063000, China
}

Zhou et al.: Time Nursing Theory on Gestational Hypertension Patients

\begin{abstract}
To analyze the application effect of time nursing theory based clinical nursing pathway on gestational hypertension patients is the main objective. A total of 85 gestational hypertension patients admitted to our hospital from January 2018 to December 2019 were enrolled in this study and divided into 2 groups according to the order of admission. 42 cases in the control group received routine nursing and 43 cases in the study group received nursing according to time nursing theory based clinical nursing pathway. The blood pressure measurement results, delivery ways, maternal and infant outcomes and negative emotions were compared between the two groups. The systolic blood pressure and diastolic blood pressure were lower in both groups after nursing $(p<0.05)$, with the systolic blood pressure and diastolic blood pressure lower in the study group than in the control group after nursing $(p<0.05)$. The study group had higher proportion of natural delivery $(p<0.05)$ and lower proportion of cesarean section than the control group $(p<0.05)$. Fetal distress, neonatal asphyxia, premature delivery and postpartum hemorrhage had lower proportion in the study group than in the control group $(\mathbf{p}<\mathbf{0 . 0 5})$. Scores of self-rating anxiety scale and self-rating depression scale were lower in both groups after nursing $(\mathbf{p}<\mathbf{0 . 0 5})$, with self-rating anxiety scale and self-rating depression scale scores lower in the study group than in the control group $(p<0.05)$. Application of time nursing theory based clinical nursing pathway in clinical nursing of gestational hypertension patients can help to control blood pressure, promote natural delivery, improve maternal and infant outcomes and alleviate patient's negative emotions.
\end{abstract}

Key words: Time nursing theory, clinical nursing pathway, gestational hypertension

Gestational hypertension is a common obstetric disease unique to pregnant women, which is often accompanied by manifestations like hypertension, proteinuria ${ }^{[1]}$. Investigations show that the disease has high incidence in the middle and late stages of pregnancy, accounting for about $10 \%$ of all pregnancies. If not properly treated, it will affect all systems and organs of the whole body, thus quite harmful to maternal and infant health and life ${ }^{[2]}$. Studies have shown that standardized treatment plus active intervention can well improve patient's physical and mental condition, delivery quality and maternal and infant outcomes ${ }^{[3]}$. Time nursing theory means to provide full range of psychological and physiological nursing services around the patient's nursing needs based on physiological rhythm of the human body ${ }^{[4]}$. Clinical nursing pathway means to establish a systematic and comprehensive nursing pathway based on patient features and disease characteristics and formulate a scientific and feasible clinical nursing schedule to guarantee high quality medical services for patients from admission to discharge ${ }^{[5]}$. This study integrates time nursing theory and clinical nursing

*Address for correspondence

E-mail: jizheng2020@163.com pathway, adopts time nursing theory based clinical nursing pathway to implement clinical nursing for gestational hypertension patients.

\section{MATERIALS AND METHODS}

\section{Clinical data:}

A total of 85 gestational hypertension patients admitted to our hospital from January 2018 to December 2019 were enrolled in this study and divided into 2 groups according to the order of admission. There are 42 cases in the control group, aged 21 40 (28.48 \pm 3.39$)$ y of age, with $31 \sim 40(35.25 \pm 1.94) \mathrm{w}$ of gestational age, including 33 cases of primiparous women and 9 cases of multiparous women respectively. Clinical grading: 14 cases, 19 cases and 9 cases of mild, moderate and severe condition. There are 43 cases in the study group, aged $20 \sim 41(28.62 \pm 3.47)$ y of age, with $30 \sim 39$ $(34.63 \pm 2.02) \mathrm{w}$ of gestation, including 36 cases of primiparous women and 7 cases of multiparous women. Clinical grading: 12 cases, 23 cases and 8 cases of mild, moderate and severe condition. In comparison of data between the two groups, the difference is not statistically 
significant ( $p>0.05)$, showing comparability.

\section{Inclusion and exclusion criteria:}

Inclusion criteria-Meet the diagnostic criteria for gestational hypertension ${ }^{[6]}$; no other complications; no history of hypertension; singleton pregnancy; establish files in the obstetrics clinic of the hospital, receive regular production inspection and pay return visits.

Exclusion criteria-Suffer from metabolic diseases (such as hyperthyroidism); suffer from heart, liver, kidney diseases; suffer from coagulation dysfunction; suffer from mental illness or have mental retardation, communication disorder, etc.; have a history of miscarriage, premature birth, etc.

\section{Nursing methods:}

Control group routine nursing, including condition monitoring, medication guidance, health education, etc. Study group-time nursing theory based clinical nursing pathway (Table 1).

\section{Observation indicators:}

Comparison of blood pressure measurement results, both systolic blood pressure and diastolic blood pressure should be measured before and after nursing.

Comparison of delivery ways, make statistics of the percentage of women with vaginal delivery, natural delivery and cesarean section in each group.

Comparison of adverse maternal and infant outcomes, adverse infant outcomes include fetal distress, neonatal asphyxia and perinatal death. Adverse maternal outcomes include premature delivery, postpartum hemorrhage and placental abruption.

Comparison of negative emotions, evaluate anxiety and depression of the two groups before and after nursing based on Self-Rating Anxiety Scale (SAS) ${ }^{[7]}$ and selfRating Depression Scale (SDS) ${ }^{[8]}$. SAS, SDS have a critical value of 50 points and 53 points, respectively. A higher score indicates more obvious anxiety and depression.

\section{Statistical processing:}

Data analysis is performed using Statistical Package for the Social Sciences (SPSS) 23.0. The measurement data conform to normal distribution and are all represented by $(\overline{\mathrm{x}} \pm \mathrm{s})$. Independent sample t-test is used for comparison between groups and paired sample t-test is used for comparison within the groups. The count data is represented by $\mathrm{n}(\%)$ and $\chi^{2}$ is used for comparison between groups. Test level $\alpha=0.05, \mathrm{p}<0.05$ indicates statistically significant difference.

\section{RESULTS AND DISCUSSION}

There is no statistically significant difference in the measurement results of systolic and diastolic blood pressure between the two groups before nursing $(p>0.05)$. The measurement results of systolic and diastolic blood pressure are lower in both groups after nursing $(\mathrm{p}<0.05)$, with measurement results of systolic and diastolic blood pressure lower in the study group than in the control group after nursing $(\mathrm{p}<0.05)$, as shown in Table 2.

There is no statistically significant difference in the proportion of vaginal delivery between the two groups $(p>0.05)$. Natural delivery has higher proportion in the study group than in the control group $(p<0.05)$ and cesarean section has lower proportion in the study group ( $\mathrm{p}<0.05)$, as shown in Table 3 .

There is no statistically significant difference between the two groups in the proportions of perinatal death and placental abruption $(\mathrm{p}>0.05)$. Fetal distress, neonatal asphyxia, premature delivery and postpartum hemorrhage have lower proportions in the study group than in the control group $(\mathrm{p}<0.05)$, as shown in Table 4 .

There is no statistically significant difference in SAS scores and SDS scores between the two groups, before nursing ( $>0.05)$. SAS scores and SDS scores are lower in both groups after nursing $(\mathrm{p}<0.05)$, with SAS scores and SDS scores lower in the study group than in the control group $(\mathrm{p}<0.05)$, as shown in Table 5 .

\section{TABLE 1: COMPARISON OF TEST RESULTS IN EACH GROUP}

\begin{tabular}{ll}
\hline Time & Nursing method \\
\hline & Set up a clinical nursing specialist team with the obstetric head nurse as the team leader, comprehensively \\
evaluate each patient's condition, physical condition, etc., formulate appropriate gestational hypertension \\
nursing pathway based on the evaluation results and prepare a table. Members of the clinical nursing \\
specialist team receive uniform training to ensure that each member can implement clinical nursing in \\
Before \\
admission \\
accordance with the nursing pathway table. The nurse marks whether content of each patient's gestational \\
hypertension nursing pathway table is implemented and check the corresponding items if it is implemented. \\
The head nurse regularly makes evaluations on nurse performance by checking the gestational hypertension \\
nursing pathway table, identifies the influencing factors that cause variation of clinical nursing pathways \\
and proposes targeted rectification measures.
\end{tabular}


$1 \mathrm{~d}$ after admission

From 2

d after admission to pre delivery

On the day of delivery

After delivery and before discharge
Ward arrangement; the ward should be tidy, clean, odour-free, soundproof, with no debris on the floor, in suitable temperature and humidity and soft light; introduce other roommates in the same ward, hospitalization environment and main medical staff to the patient/family; introduce the auxiliary inspection plan in detail, explain the purpose of each inspection and assist the patient in completing each inspection.

Health education; at the best time of the patient's mental state, find the right time, create an environment suitable for conversation and introduce the patient/family to the knowledge of gestational hypertension, including the mechanism of occurrence, treatment plan, etc., emphasize the necessity and importance of active cooperation in the treatment to help patient develop self-care consciousness. The health education should be patiently performed with language concise, vivid and easy to understand.

Psychological nursing: Give psychological nursing every day from 19:00 to 21:00, establish a trusted nursepatient relationship with the patient/family, inform the patient of the relationship between mental state and gestational hypertension, choose appropriate psychological intervention according to the patient's personality characteristics and psychological state, etc. provide psychological support and guidance in a timely manner. Work and rest guidance: It is advisable to take the left lateral position when sleeping, pay attention to regular work and rest with daily rest time $\geq 10 \mathrm{~h}$, avoid the peak blood pressure period in regular activities during the day, avoid excitatory activities before going to bed, soak feet in warm water.

Diet nursing: According to the gastric emptying time, small intestine absorption time, etc., work with the hospital nutritionist to adjust the patient's diet. Patients should have more meals a day but less food at each. Diet nature (normal diet, soft food, etc.) should be taken into account in deciding the specific number of meals. In addition, limit salt, scientifically supplement protein, vitamins, calcium, etc.

Life guidance: According to the general laws and individual differences of human physiological changes, etc., instruct patients to rest for a while after waking up in the morning and then perform daily activities such as washing face and brushing teeth. Patients should drink moderate amounts of warm water on an empty stomach; wash face, rinse mouth and soak their feet at night before going to bed. Medical staff should try their best to complete relevant treatment and nursing operations before the patient goes to bed.

Condition observation: According to the general laws of human physiological changes, monitor and record various vital signs at the best time. For example, blood pressure should be measured at 10:00 and 18:00 and body temperature should be measured after patient wakes up in the morning and before patient goes to bed at night. Instruct and teach patients/family how to measure blood pressure.

Medication guidance: According to each patient's medication regimen, blood pressure circadian rhythm, etc., determine the patient's medication time and dosage accordingly and increase the medication dosage reasonably during the high-risk period of gestational hypertension to keep the blood concentration at the peak. Nurses and family members of patients should urge patients to correctly implement medical instructions and take correct dosage of drugs at the correct time according to correct medication method.

Accurate and whole course nursing should be provided according to the patient's delivery way (vaginal delivery, cesarean section, etc.). For example, for vaginal delivery, corresponding nursing should be provided according to the delivery process and patient's nursing needs; for cesarean section, visit in advance, introduce the patient to the related contents of cesarean section, etc. (including fetal heart rate monitoring, blood pressure monitoring, psychological nursing, etc.).

Strengthen condition observation after delivery, implement psychological nursing, life guidance, etc. and teach patients correct breastfeeding method and uterine massage.

After discharge from the hospital, keep in touch with the patient through WeChat, phone, etc., and contact the patient by phone 1 mo after delivery so that subsequent visit is made.

TABLE 2: COMPARISON OF BLOOD PRESSURE MEASUREMENT RESULTS ( $\overline{\mathbf{x}} \pm \mathbf{s} \mathbf{m m H g})$

\begin{tabular}{lccccc}
\hline \multirow{2}{*}{ Group } & \multirow{2}{*}{ Number of cases } & \multicolumn{2}{c}{ Systolic blood pressure } & \multicolumn{2}{c}{ Diastolic blood pressure } \\
\cline { 3 - 6 } & & Before nursing & After nursing & Before nursing & After nursing \\
\hline Control group & 42 & $151.54 \pm 11.12$ & $134.53 \pm 12.18^{(1)}$ & $106.59 \pm 8.92$ & $90.12 \pm 7.15^{(1)}$ \\
Study group & 43 & $152.42 \pm 11.23$ & $123.36 \pm 10.54^{(1)}$ & $105.83 \pm 9.14$ & $84.75 \pm 6.28^{(1)}$ \\
$\mathrm{t}$ & - & 0.363 & 4.525 & 0.388 & 3.681 \\
$\mathrm{p}$ & - & 0.718 & 0.000 & 0.699 & 0.000 \\
\hline
\end{tabular}

Note: Compared with the same group before nursing ${ }^{(1)}, p<0.05$ 
TABLE 3: COMPARISON OF DELIVERY WAYS CASE (\%)

\begin{tabular}{lcccc}
\hline Group & Number of cases & Natural delivery & Vaginal delivery & Cesarean section \\
\hline Control group & 42 & $12(28.57)$ & $10(23.81)$ & $20(47.62)$ \\
Study group & 43 & $22(51.16)$ & $10(23.26)$ & $11(25.58)$ \\
$\chi^{2}$ & - & 4.518 & 0.004 & 4.454 \\
$\mathrm{p}$ & - & 0.034 & 0.952 & 0.035 \\
\hline
\end{tabular}

TABLE 4: COMPARISON OF NEGATIVE EMOTIONS ( $\overline{\mathbf{x}} \pm \mathbf{s}$, POINT)

\begin{tabular}{lccccc}
\hline \multirow{2}{*}{ Group } & \multirow{2}{*}{ Number of cases } & \multicolumn{2}{c}{ SAS } & \multicolumn{2}{c}{ SDS } \\
\cline { 3 - 6 } & & Before nursing & After nursing & Before nursing & After nursing \\
\hline Control group & 42 & $54.63 \pm 7.98$ & $49.55 \pm 6.46^{(1)}$ & $53.75 \pm 8.69$ & $47.48 \pm 7.52^{(1)}$ \\
Study group & 43 & $55.24 \pm 8.12$ & $41.42 \pm 5.28^{(1)}$ & $54.37 \pm 9.15$ & $40.45 \pm 6.13^{(1)}$ \\
$\mathrm{t}$ & - & 0.349 & 6.360 & 0.320 & 4.729 \\
$\mathrm{p}$ & - & 0.728 & 0.000 & 0.750 & 0.000 \\
\hline
\end{tabular}

Note: Compared with the same group before nursing ${ }^{(1)}, p<0.05$

TABLE 5: COMPARISON OF MATERNAL AND INFANT OUTCOMES CASE (\%)

\begin{tabular}{|c|c|c|c|c|c|c|c|}
\hline \multirow[b]{2}{*}{ Group } & \multirow{2}{*}{$\begin{array}{c}\text { Number of } \\
\text { cases }\end{array}$} & \multicolumn{3}{|c|}{ Infant outcome } & \multicolumn{3}{|c|}{ Maternal outcome } \\
\hline & & Fetal distress & $\begin{array}{l}\text { Neonatal } \\
\text { asphyxia }\end{array}$ & $\begin{array}{c}\text { Perinatal } \\
\text { death }\end{array}$ & $\begin{array}{c}\text { Premature } \\
\text { delivery }\end{array}$ & $\begin{array}{l}\text { Postpartum } \\
\text { hemorrhage }\end{array}$ & $\begin{array}{l}\text { Placental } \\
\text { abruption }\end{array}$ \\
\hline Control group & 42 & $11(26.19)$ & $10(23.81)$ & $2(4.76)$ & $8(19.05)$ & $12(28.57)$ & $5(11.90)$ \\
\hline Study Group & 43 & $4(9.30)$ & $3(6.98)$ & $0(0.00)$ & $2(4.65)$ & $3(6.98)$ & $2(4.65)$ \\
\hline$\chi^{2}$ & - & 4.170 & 4.647 & 2.097 & 4.242 & 6.818 & 1.479 \\
\hline $\mathrm{p}$ & - & 0.041 & 0.031 & 0.148 & 0.039 & 0.009 & 0.224 \\
\hline
\end{tabular}

Abnormal increase in blood pressure is the main manifestation of gestational hypertension, so scientific and effective blood pressure reduction and control measures should be taken in time. While standardizing the treatment, this study provides medication guidance based on the circadian rhythm of blood pressure, so that patients can take medicine correctly according to correct medication method at the right time to maximize the effectiveness of drug therapy. At the same time, guided by the general law of human physiological changes and other relevant time nursing theories, the specific content of clinical nursing pathway table should be implemented at the right time to provide patients with more comprehensive and scientific nursing services, significantly control blood pressure and lower blood pressure $^{[9]}$. Liuzheng ${ }^{[10]}$ shows that high-quality nursing for patients with gestational hypertension can effectively control their blood pressure. The results of this study indicate that the systolic and diastolic blood pressure measurement results are lower in the study group than in the control group after nursing, suggesting that application of time nursing theory based clinical nursing pathway in clinical nursing of gestational hypertension patients can control and reduce blood pressure more effectively.
Studies have shown that nursing intervention among gestational hypertension patients can significantly improve the success rate of natural delivery ${ }^{[11]}$. This study cleverly uses time nursing theory based clinical nursing pathway to guide clinical nursing. On the one hand, time factors are linked to nursing practice, so that time nursing theory provides basis for the implementation of specific measures in clinical nursing pathway, thus guaranteeing efficiency, predictability of nursing intervention. On the other hand, clinical nursing pathway table has holistic and systematic advantages, which establishes a systematic and comprehensive nursing pathway based on patient features and characteristics of gestational hypertension, etc., formulates a scientific and feasible clinical nursing schedule to guarantee high-quality medical services for patients from admission to discharge and better serve the treatment of gestational hypertension ${ }^{[12]}$. This study integrates time nursing theory and clinical nursing pathway and combines the advantages of both, thus producing a positive impact on natural delivery. The results indicate that the study group has higher proportion of natural delivery and lower proportion of cesarean section than the control group, suggesting that application of time nursing theory based clinical 
nursing pathway to clinical nursing of gestational hypertension patients can promote natural delivery and reduce the proportion of cesarean section.

Clinical nursing pathway emphasizes patient centered service concept in provision of thoughtful, meticulous and comprehensive nursing services. In the specific implementation process of this study, guided by time nursing theory, specific implementation time of contents of the clinical nursing pathway table is reasonably allocated. By nursing according to different diseases and nursing according to different time, quality of clinical nursing is significantly improved with purpose of adjuvant treatment achieved, so that occurrence of risk events is effectively reduced, thus ultimately improving the maternal and infant outcome. Guixia ${ }^{[13]}$ shows that nursing interventions for patients with gestational hypertension can significantly improve maternal and infant outcomes. The results of this study indicate that fetal distress, neonatal asphyxia, premature delivery and postpartum hemorrhage, have lower proportions in the study group than in the control group, suggesting that application of time nursing theory based clinical nursing pathway in clinical nursing of gestational hypertension patients can improve maternal and infant outcomes.

Gestational hypertension is a psychosomatic disease. Most patients lack sufficient knowledge of the disease and worry that the disease itself is unfavorable for the fetus, thus easily developing negative psychology. Therefore, active treatment should also take patient's psychological nursing into account ${ }^{[14]}$. Zhanhui et al. ${ }^{[15]}$ demonstrates that psychological nursing has positive significance in clinical nursing of gestational hypertension patients. This study selects the time period with increased mental activity (19:00 21:00 according to the time nursing theory) to implement psychological nursing and then selects appropriate psychological support measures according to the patient's personality characteristics and psychological state, so as to effectively alleviate the patient's unhealthy emotions. The results show that SAS and SDS scores are lower in the study group than in the control group after nursing, suggesting that application of time nursing theorybased clinical nursing pathway in clinical nursing of gestational hypertension patients can improve the patient's emotional state and significantly relieve anxiety and depression.
To sum up, application of time nursing theory based clinical nursing pathway in clinical nursing of gestational hypertension patients can help to control blood pressure, promote natural delivery, improve maternal and infant outcomes and alleviate patient's negative emotions.

\section{Conflicts of interest:}

The authors declared no conflict of interest.

\section{REFERENCES}

1. Jiang HQ, Chen H, Song HY, Qian NF, Zhang S, Wang A. Establishment and analysis of risk prediction model for gestational hypertension. Acad J Chin PLA Med Sch 2018;39(10):844-9.

2. Valensise H, Novelli GP, Vasapollo B, Di Ruzza G, Romanini ME, Marchei M, et al. Maternal diastolic dysfunction and left ventricular geometry in gestational hypertension. Hypertension 2001;37(5):1209-15.

3. Wang YL. Application of moderate aerobic exercise intervention combined with meticulous nursing mode in patients with gestational hypertension. Chin Rem Clin 2018;18:125-7.

4. Wu LY. Application of clinical nursing pathway in gestational diabetes mellitus complicated with gestational hypertension. Med Inf 2019;32(10):181-3.

5. Zhao YX, Wang SJ, Yang XP. Application of clinical nursing pathway in health education for patients with level 2 hypertension. West J Chin Med 2018;31(7):118-20.

6. Li M, Bai LH, Wang Y. Study on the incidence of white coat hypertension in early pregnancy of diabetic women and its relationship with hypertension in pregnancy. Chin J Lab Diagn 2021;25(1):49-53.

7. Li WL, Cai LK. Analysis on the effect of clinical nursing intervention in pregnant patients with hypertension and influence for sleep quality. World J Sleep Med 2020;7(4):675-6.

8. Zung WW. A self-rating depression scale. Arch Gen Psychiatry 1965;12(1):63-70.

9. Gofton EN, Capewell V, Natale R, Gratton RJ. Obstetrical intervention rates and maternal and neonatal outcomes of women with gestational hypertension. Am J Obstet Gynecol 2001;185(4):798-803.

10. Wang LZ. Effect of high quality nursing on postpartum hemorrhage of pregnant women with pregnancy induced hypertension. Pract J Card Cereb Pneumal Vasc Dis 2018;26:399-401.

11. Ning XH. Effect of nursing intervention on the natural delivery success rate and quality of life of patients with pregnancy-induced hypertension syndrome. Harbin Med J 2018;38(6):592-3.

12. Huang LM, Zhou W. Effect of clinical nursing pathway on gestational hypertension patients. China Pract Med 2017;12(16):165-6.

13. Tan GX. Effect of comprehensive nursing intervention model on negative emotions and maternal and infant outcomes of gestational hypertension patients. Henan Med Res 2018;27(22):4199-200. 
14. Wang ZX, Cao WP. A qualitative study on the psychological needs of gestational hypertension patients. Chin Gen Pract Nurs 2019;17(9):1119-22.

15. Wang ZH, Jiang YY, Zhang LY. Clinical application of psychological nursing in patients with gestational hypertension-induced intrauterine growth retardation. Int $\mathbf{J}$ Nurs 2018;37(8):1113-5.
This is an open access article distributed under the terms of the Creative Commons Attribution-NonCommercial-ShareAlike 3.0 License, which allows others to remix, tweak, and build upon the work non-commercially, as long as the author is credited and the new creations are licensed under the identical terms

This article was originally published in a special issue, "Diagnostic and Therapeutic Advances in Biomedical Research and Pharmaceutical Sciences"

Indian J Pharm Sci 2021:83(5)Spl Issue "243-248" 